\title{
Development of a 3D-printed testicular cancer model for testicular examination education
}

Rebecca J. Power, $\mathrm{MSc}^{1}$; Jason Hearn, $\mathrm{MHSc}^{1}$; Charlie J. Gillis, MD ${ }^{1}$; David Harvey, MD, FRCSC $^{2}$; Christopher French, MD, FRCSC ${ }^{2}$; Michael Organ, MD, FRCSC ${ }^{2}$

${ }^{1}$ Faculty of Medicine, Memorial University of Newfoundland, St. John's, NL, Canada; ${ }^{2}$ Department of Urology, Discipline of Surgery, Faculty of Medicine, Memorial University of Newfoundland, St. John’s, NL, Canada

Support: This study was supported by the Strategy for Patient Outreach-Oriented Research-Canadian Institutes of Health Research.

Cite as: Power RJ, Hearn J, Gillis CJ, et al. Development of a 3D-printed testicular cancer model for testicular examination education. Can Urol Assoc J 2020 September 28; Epub ahead of print. http://dx.doi.org/10.5489/cuaj.6675

Published online September 28, 2020

$* * *$

\section{Abstract}

Introduction: Testicular cancer is the most commonly diagnosed malignancy in young males. Testicular examination is a non-invasive and inexpensive means of detecting testicular cancer at an early stage. In this project, a set of 3D-printed models was developed to facilitate teaching testicular examination and improving understanding of testicular malignancies among patients and medical learners.

Methods: Five scrotum models were designed: a control model with healthy testes, and four models containing a healthy testicle and a testicle with an endophytic mass of varying size. The anatomy, texture, and composition of the 3D-printed models were refined using an iterative process between the design team and urologists. The completed models were assessed by six urologists, two urology nurse practitioners, and 32 medical learners. Participants were asked to inspect and palpate each model, and to provide feedback using a five-point Likert scale.

Results: Clinicians reported that the models enabled accurate simulation of a testicular examination involving both healthy and pathological testes $(\overline{\mathrm{x}}=4.3 \pm 1.0)$. They agreed that the models would be useful teaching tools for both medical learners $(\bar{x}=4.8 \pm 0.5)$ and patients $(\bar{x}=4.8 \pm 0.7)$. Following an educational session with the models, medical learners reported improvements in confidence and skill in performing a testicular examination.

Conclusions: 3D-printed models can effectively simulate palpation of both healthy and pathological testes. The developed models have the potential to be a useful adjunct in teaching testicular examination and in demonstrating abnormal findings that require further investigation. 


\section{Introduction}

Testicular cancer is the most commonly diagnosed malignancy in men aged 15-44 years living in North America. ${ }^{1}$ Testicular cancer is highly amenable to treatment when caught at an early stage ${ }^{2}$ with a five-year survival approaching $100 \%$ in patients diagnosed at stage $\mathrm{I}^{3}{ }^{2}$ In contrast, patients who miss the early window and present with a stage IV metastatic diagnosis have a fiveyear survival of $74 \% .{ }^{3}$ Early detection and management of cancerous testicular lesions is thus critical in optimizing patient outcomes.

As testicular cancer often presents as a painless mass in the scrotum and/or testicular swelling, ${ }^{4-6}$ testicular examination is a non-invasive and simple test to identify such pathologic findings at an early stage. However, past research has shown that only $5 \%$ of medical students beginning their clinical clerkship training, ${ }^{7}$ as well as only $36 \%$ of pediatrics residents, ${ }^{8}$ identify as comfortable with their testicular examination skills. Accumulating evidence also suggests potential benefits of testicular self-examination (TSE) as a screening test given its privacy and convenience; 9,10 however, previous surveys amongst male college students report that only $41 \%$ are taught TSE and only 8\% perform the examination with regularity. Such results highlight an educational gap amongst both medical learners and patients, as well as a need for novel tools that will facilitate such education.

One technology that enables the development of high-fidelity educational tools is 3D printing. This technology has the distinct advantage of demonstrating spatial relations between anatomically-accurate structures, making it well-suited for use in both medical training and patient education. ${ }^{11-13}$ The use of 3D-printed models as an adjunct to existing techniques has been found to be preferred by patients, as well as beneficial in improving their understanding of relevant anatomy. ${ }^{14-18}$ Similar advantages have been demonstrated in medical education, where 3D-printed models have been effective in improving anatomy and clinical skills curricula. ${ }^{19-22}$ Despite the reported accuracy and educational benefits of 3D-printed anatomical models, no previous study (to the authors' knowledge) has investigated 3D printing as a means of improving testicular examination education. To this end, the presented study describes the development of a set of 3D-printed models designed for the purposes of teaching testicular examination and improving understanding of testicular malignancies amongst patients and medical learners.

\section{Methods}

A multidisciplinary team comprising urologists, engineers, and medical students collaborated to conceptualize a set of 3D-printed testicular cancer models. Five models were designed, with each simulated scrotum containing either a) two healthy testicles, or b) one healthy testicle and one testicle with an endophytic lesion of varying size. The endophytic lesions would be placed at varying locations within the pathologic testicle and would range in size from $5 \%$ to $60 \%$ of the total size of the testicle. Moreover, larger testicle models were used for bigger masses, simulating the testicular swelling that is often concurrent with testicular lesions. 
An initial prototype was designed and developed to simulate a normal human scrotum, testes, and epididymides. An iterative design process between the design team and urologists allowed for extensive refinement of the models in terms of anatomical accuracy. The urologists were presented with three printing materials - Dragon Skin 10 NV, Dragon Skin Fx-Pro, and Smooth-On Ecoflex 00-30 - and asked to select the printing materials that they felt best simulated the texture and consistency of a scrotum, testicle, and epididymis. The design team used the urologist feedback to further optimize the developed model, and this process was repeated until the urologists agreed that the model accurately simulated a human scrotum both on inspection and palpation (Figure 1).

Next, a similar process was used to develop a series of models containing a cancerous testicle. Testicular masses were simulated by first casting an irregular shape in a denser material than that used for the testicle. This irregular mass was then suspended in the mold used for the testicle during the casting process. This enabled an irregular mass to be placed within the testicle and to be appreciated on palpation. The urologists were presented with individual samples casted in thermoplastic elastomer and polylactic acid polymer and were asked to select which material best simulated a testicular malignancy. The size, texture, and location of the masses were also guided by frequent input from the urologists. An iterative process was repeated until the urologists were satisfied that the models accurately simulated palpation of a cancerous testicle (Figure 1). All aspects of the model were designed in OpenJSCAD and finalized using MeshMixer. All models were casted using a Prusa MKS3S printer.

Once the set of testicular cancer models had been developed, two separate sessions were held to ascertain feedback from both clinicians and medical learners. In the first session, a group of urologists and urology nurse practitioners was asked to visually inspect and palpate the developed models. In a second session, first- and second-year medical students were provided with a brief tutorial on testicular examination by a staff urologist. Of the medical learners, the first-year medical students had not yet received formal urological education as part of their undergraduate medical curriculum, while the second-year medical students had received one hour of didactic teaching on testicular cancer and two hours on urologic examination skills. These learners were then asked to practice their examination skills using the developed models.

After using each model, participants in both sessions were asked to complete a survey in which they were asked to rate their agreement with several statements using a five-point Likert scale. Items on the clinician survey primarily focused on the anatomical accuracy of the developed models, the usefulness of the models in simulating a testicular examination, and the overall applicability of the models as teaching tools. The survey for medical learners featured additional items relating to the pre- and post-session levels of both skill and confidence in performing a testicular examination. Clinicians and medical learners were also asked to select, from a list of five potential applications (e.g. medical student training, resident training, nurse practitioner training, family physician training, patient education), all of the purposes for which 
they felt the models would be beneficial. Lastly, both surveys included a section for participants to provide qualitative feedback relating to areas for improvement and the potential clinical applicability of the developed models.

\section{Results}

Through the multidisciplinary design process, it was determined that Smooth-On Ecoflex 00-30 silicone was the most anatomical representative material for the model's scrotum and testicle texture. Similar evaluation of materials for the testicular pathologies concluded that polylactic acid polymer was the most anatomically representative material (Figure 2).

A total of six urologists and two urology nurse practitioners participated in the study. Responses from the surveyed urologists and urology nurse practitioners are summarized in Table 1. All surveyed participants agreed that the developed models would be useful teaching tools for both medical learners and patients. They also agreed that these models would be beneficial for resident, nurse practitioner, and family physician training. Respondents felt that the models enabled accurate simulation of a testicular examination. All participants agreed that the models effectively simulated palpation of healthy and pathologic testes. Qualitative feedback concluded that the majority would use these models as a teaching aid for both patients and medical learners. In addition, all agreed that they would incorporate these models in some way into their practice. In terms of areas for improvement, respondents suggested that the epididymis could be more prominent to more accurately simulate palpation. These recommendations were used to further optimize the anatomical accuracy of the testicular cancer model (Figure 1, Figure 2. C).

Survey data were collected from 32 medical learners. Of the 32 learners, 26 were firstyear medical students and 6 were second-year medical students. The results of the medical learner survey are summarized in Table 2. The proportion of medical learners identifying as confident in performing a testicular examination increased from a pre-session value of $6.3 \%$ to $84.4 \%$ following the session (Table 2). A similar effect was demonstrated in the proportion of learners claiming that they possessed the skills to perform a testicular examination, which increased from $6.3 \%$ to $100 \%$ (Table 2 ). The majority of medical learners felt that the use of the testicular models would be helpful in the current medical school curriculum. In addition, most medical learners believed that these models would be beneficial for medical student training $(96.9 \%)$ and patient education $(90.6 \%)$. The majority of medical learners stated that these models would be useful for resident training $(75.0 \%)$, nurse practitioner training $(84.4 \%)$, and family physician training $(75.0 \%)$. Medical learner qualitative feedback concluded that the testicular models were helpful in identifying a testicular mass on palpation, practising palpation technique, and differentiating between a pathologic and healthy testicle.

\section{Discussion}

The presented 3D-printed testicular models have the potential to improve testicular cancer education for medical learners and patients. From the survey of medical learners, the models 
were shown to increase both skill and confidence relating to the performance of a testicular examination. Both medical learners and clinicians agreed that these models would be a beneficial addition to the existing medical learner urology curriculum. Learning to perform a genitourinary examination on actual patients can be uncomfortable for both the patient and the learner. Alternative teaching modalities can potentially relieve anxiety associated with the standard apprenticeship method of teaching urologic examination skills, as well as create a safe environment for learners to refine their skills. ${ }^{7,23,24}$ A study by Kaplan et al. stated that, after medical students were given an intensive examination skills course using a standardized patient, $90.3 \%$ of students reported being significantly more comfortable with performing a testicular examination. These medical students reported that learning a testicular examination was one of the most useful urologic skills to learn in a standardized environment. ${ }^{7}$ Medical learners have also previously reported a preference for practising examination skills on male anatomical models rather than standardized patients. ${ }^{23}$ In fact, one study found crude handmade models simulating testicular swelling pathologies to be beneficial in teaching urologic examination skills to medical students. ${ }^{24}$ As using a standardized patient can be costly and logistically burdensome, simulated education tools offer a convenient alternative that may improve testicular examination education. $^{23}$

The survey data also suggested that the developed models would be beneficial in educating patients about TSE and testicular malignancies in general. It has been previously reported that there is a significant lack of education in the general public surrounding testicular cancer and TSE. ${ }^{25-27}$ Misinformation relating to urologic cancers can be easily spread through social media. ${ }^{25,26}$ A recent publication by Yeo et al. suggested that, with the increase in patients acquiring information about testicular cancer from sources that are not validated or credible, direct patient education has become even more important. ${ }^{28}$ Directing patients towards validated testicular cancer education programs is beneficial for patient-physician communication, as well as overall patient understanding. The testicular cancer model described in this study can serve as an adjunct for patient education and facilitate discussion regarding the benefits of screening and the risk of testicular malignancy.

Currently, the number of testicular cancer models available on the market is limited. The available models are expensive and fail to accurately show disease progression. From a review of the current products available on the market, a progressive set of five testicular cancer models would cost approximately $\$ 875$ Canadian Dollars (CAD), while a male pelvic trainer would cost approximately $\$ 3700 \mathrm{CAD}$. The use of 3D printing offers a unique solution to the high cost of existing models. The material cost of the five progressive testicular cancer models presented in this study was approximately \$13 CAD (\$10 of Smooth-On Ecoflex 00-30 silicone and \$3 CAD of polylactic acid polymer). That is a direct cost savings of $\$ 862 \mathrm{CAD}$. In addition, many universities and public libraries now offer 3D printing services that could be used to print the developed models at a low cost. Accordingly, 3D-printed technology allows for anatomically 
accurate models to be made at a fraction of the cost of existing models; thus, mitigating the financial barriers currently associated with quality testicular cancer education.

Using 3D-printed models as an adjunct to existing techniques is rated as a highly valuable learning tool for patients, as well as beneficial in improving their understanding of relevant anatomy and surgical complications. ${ }^{14-18}$ In addition, truly patient-informed decision-making is predicated on a basic understanding of pathology and anatomy, a process that can be facilitated with various patient education aids. This technology has been shown to be a successful means of educating patients in urology. ${ }^{17,29,30}$ In a recent systematic review performed by Lupulescu and Sun, 27 studies analyzed the use of 3D-printed technologies for preoperative surgical planning and patient education in renal surgery. This review found that patient-specific 3D-printed models were useful for educating patients and their families on renal surgery, with high reported levels of satisfaction when using 3D-printed models. ${ }^{11}$

Testicular cancer commonly presents with symptoms that are recognizable by patients; thus, it is important that the general population receives proper education on how to complete a TSE. Despite the potential benefits and relative ease of TSE, it should be noted that its use as a broad screening tool is debated in the current literature. Currently, neither the Canadian Urological Association nor the American Urological Association have published guidelines related to testicular cancer screening and TSE. The United States Preventative Task Force recommends against testicular cancer screening in asymptomatic males due to a lack of evidence demonstrating a benefit and a potential association with increased anxiety related to a falsepositive result. ${ }^{31,32}$ They do, however, recommend TSE in the context of high-risk individuals, such as those with cryptorchidism or a positive family history. ${ }^{31,32}$ The Society for Adolescent Health and Medicine recommends TSE, as they state that it identifies several risk factors for testicular cancer. ${ }^{33}$ It has also been found that TSE is associated with improved education and increased comfort amongst young adult males receiving a genital examination. ${ }^{33}$ Despite the current discrepancies between various organizations, it is generally agreed that there is a significant proportion of men in the general population that are unaware of the causes and symptoms of testicular cancer. Moreover, the unclear benefit of TSE is likely attributable to a current lack of large population studies assessing the effect of this screening method.

The results of this study must be viewed in light of its limitations. The information collected in this study was ascertained from a single institution in a relatively small cohort. The large increase seen in medical learner skill and confidence in performing testicular examinations could be attributed to some external factors. The educational session was the first time that many of the students were exposed to testicular cancer education; thus, pre- and post-session selfreported outcomes were likely not the best metrics for quantifying educational benefit. As a caveat to this limitation, the second-year medical students participating in the session qualitatively noted that the 3D-printed models would be a useful adjunct to the existing urologic clinical skills curriculum. 
Additional research is warranted for clinical validation of the developed 3D-printed models. Future work could include ascertaining feedback from a larger cohort of respondents, including members of the general public and high-risk patients. In order to demonstrate educational utility, it would also be beneficial to incorporate these models into the existing urology curriculum and to assess the associated educational effect using an interventional study design. This could be accomplished by comparing performance on standardized clinical examinations between students with and without exposure to the models as part of their urology curriculum. Similar models replicating other testicular and scrotal pathologies such as epididymal cyst, testicular torsion and hydrocele could also be designed and developed using an iterative design process similar to the methodology outlined in this study.

\section{Conclusions}

This study describes the development and preliminary validation of 3D-printed urological models that may fill existing gaps in patient and medical learner education. 3D-printed models can simulate anatomical structures in a low-cost and effective manner. In the field of urology, this technology presents a unique opportunity to develop and produce educational models that can maintain a high level of fidelity at a low cost. In this study, a set of testicular cancer education models was developed. These models were well-accepted by surveyed urology practitioners and medical learners. The models were also shown to improve both medical learner skill and confidence in performing a testicular examination. The developed 3D-printed models may enable urologists and family physicians to better educate their patients, as well as assist medical learners in developing testicular examination skills. 


\section{References}

1. Trabert B, Chen J, Devesa SS, Bray F, McGlynn KA. International patterns and trends in testicular cancer incidence, overall and by histologic subtype, 1973-2007. Andrology. 2015;3(1):4-12.

2. Cheng L, Albers P, Berney DM, et al. Testicular cancer. Nat Rev Dis Primers. 2018;4(1):29.

3. Miller KD, Nogueira L, Mariotto AB, et al. Cancer treatment and survivorship statistics, 2019. CA Cancer J Clin. 2019;69(5):363-385.

4. Germà-Lluch JR, Garcia del Muro X, Maroto $\mathrm{P}$, et al. Clinical pattern and therapeutic results achieved in 1490 patients with germ-cell tumours of the testis: the experience of the Spanish Germ-Cell Cancer Group (GG). Eur Urol. 2002;42(6):553-562; discussion 562-563.

5. Wynd CA. Testicular self-examination in young adult men. J Nurs Scholarsh. 2002;34(3):251-255.

6. Khadra A, Oakeshott P. Pilot study of testicular cancer awareness and testicular selfexamination in men attending two South London general practices. Fam Pract. 2002;19(3):294-296.

7. Kaplan AG, Kolla SB, Gamboa AJR, et al. Preliminary evaluation of a genitourinary skills training curriculum for medical students. J Urol. 2009;182(2):668-673.

8. Brenner JS, Hergenroeder AC, Kozinetz CA, Kelder SH. Teaching testicular selfexamination: education and practices in pediatric residents. Pediatrics. 2003;111(3):e239-e244.

9. Aberger M, Wilson B, Holzbeierlein JM, Griebling TL, Nangia AK. Testicular selfexamination and testicular cancer: a cost-utility analysis. Cancer Med. 2014;3(6):16291634.

10. Rovito MJ, Leone JE, Cavayero CT. “Off-Label” Usage of Testicular Self-Examination (TSE): Benefits Beyond Cancer Detection. Am J Mens Health. 2018;12(3):505-513.

11. Lupulescu C, Sun Z. A Systematic Review of the Clinical Value and Applications of Three-Dimensional Printing in Renal Surgery. J Clin Med Res. 2019;8(7). doi: $10.3390 / \mathrm{jcm} 8070990$

12. Tejo-Otero A, Buj-Corral I, Fenollosa-Artés F. 3D Printing in Medicine for Preoperative Surgical Planning: A Review. Ann Biomed Eng. 2020;48(2):536-555.

13. Shafiee A, Atala A. Printing Technologies for Medical Applications. Trends Mol Med. 2016;22(3):254-265.

14. Zhuang Y-D, Zhou M-C, Liu S-C, Wu J-F, Wang R, Chen C-M. Effectiveness of personalized 3D printed models for patient education in degenerative lumbar disease. Patient Educ Couns. 2019;102(10):1875-1881.

15. Yang T, Tan T, Yang J, et al. The impact of using three-dimensional printed liver models for patient education. J Int Med Res. 2018;46(4):1570-1578.

16. van de Belt TH, Nijmeijer H, Grim D, et al. Patient-Specific Actual-Size ThreeDimensional Printed Models for Patient Education in Glioma Treatment: First Experiences. World Neurosurg. 2018;117:e99-e105.

17. Schmit C, Matsumoto J, Yost K, et al. Impact of a 3D printed model on patients' 
understanding of renal cryoablation: a prospective pilot study. Abdom Radiol (NY). 2019;44(1):304-309.

18. Teishima J, Takayama Y, Iwaguro S, et al. Usefulness of personalized threedimensional printed model on the satisfaction of preoperative education for patients undergoing robot-assisted partial nephrectomy and their families. Int Urol Nephrol. 2018;50(6):1061-1066.

19. Lim KHA, Loo ZY, Goldie SJ, Adams JW, McMenamin PG. Use of 3D printed models in medical education: A randomized control trial comparing 3D prints versus cadaveric materials for learning external cardiac anatomy. Anat Sci Educ. 2016;9(3):213-221.

20. Chen $\mathrm{S}, \mathrm{Pan} \mathrm{Z}, \mathrm{Wu} \mathrm{Y}$, et al. The role of three-dimensional printed models of skull in anatomy education: a randomized controlled trail. Sci Rep. 2017;7(1):575.

21. Gillis C, Harvey D, Bishop N, Walsh G, Dubrowski A. Male catheter insertion simulation using a low-fidelity 3D-printed model in undergraduate medical learners. DALHOUSIE MEDICAL JOURNAL. 2019;45(2). doi:10.15273/dmj.vol45no2.8998

22. Ryan JR, Almefty KK, Nakaji P, Frakes DH. Cerebral Aneurysm Clipping Surgery Simulation Using Patient-Specific 3D Printing and Silicone Casting. World Neurosurg. 2016;88:175-181.

23. Taylor JS, Dube CE, Pipas CF, Fuller BK, Lavallee LK, Rosen R. Teaching the testicular exam: a model curriculum from "A" to "Zack." Fam Med. 2004;36(3):209213.

24. Sarmah PB, Sarmah BD, Ibrahim H, Panting J. Making models to simulate testicular swellings. Clin Teach. 2017;14(6):432-436.

25. Alsyouf M, Stokes P, Hur D, Amasyali A, Ruckle H, Hu B. "Fake News" in urology: evaluating the accuracy of articles shared on social media in genitourinary malignancies. BJU International. 2019;124(4):701-706. doi:10.1111/bju.14787

26. Loeb S, Taylor J, Borin JF, et al. Fake News: Spread of Misinformation about Urological Conditions on Social Media. European Urology Focus. 2019. doi:10.1016/j.euf.2019.11.011

27. Thornton CP. Best Practice in Teaching Male Adolescents and Young Men to Perform Testicular Self-Examinations: A Review. J Pediatr Health Care. 2016;30(6):518-527.

28. Yeo S, Eigl B, Ingledew P-A. A fountain of knowledge? The quality of online resources for testicular cancer patients. Can Urol Assoc J. March 2020. doi:10.5489/cuaj.6154

29. Wake N, Rosenkrantz AB, Huang R, et al. Patient-specific 3D printed and augmented reality kidney and prostate cancer models: impact on patient education. $3 D$ Print Med. 2019;5(1):4.

30. Bernhard J-C, Isotani S, Matsugasumi T, et al. Personalized 3D printed model of kidney and tumor anatomy: a useful tool for patient education. World J Urol. 2016;34(3):337345.

31. U.S. Preventive Services Task Force. Screening for testicular cancer: U.S. Preventive Services Task Force reaffirmation recommendation statement. Ann Intern Med. 2011;154(7):483-486.

32. Akar SZ, Bebiş H. Evaluation of the effectiveness of testicular cancer and testicular self-examination training for patient care personnel: intervention study. Health Educ Res. 2014;29(6):966-976. 
33. The Male Genital Examination: A Position Paper of the Society for Adolescent Health and Medicine. Journal of Adolescent Health. 2012;50(4):424-425.

doi:10.1016/j.jadohealth.2012.01.002

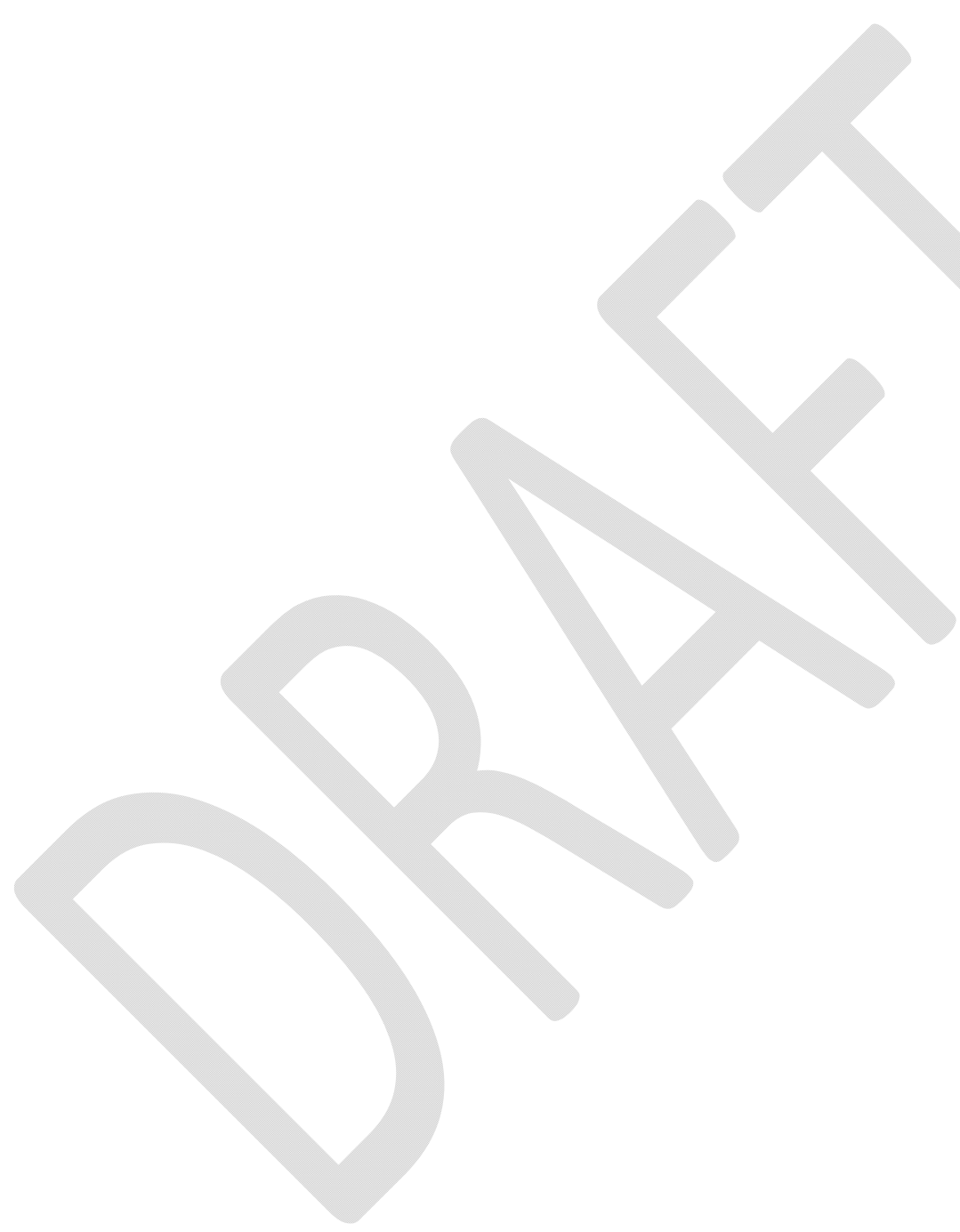




\section{Figures and Tables}

Fig. 1. Iterative design process used for the design and completion of the 3D-printed testicular cancer models. ${ }^{*}$ Feedback sessions were held with urologists, nurse practitioners and medical students that were not a part of the research team.

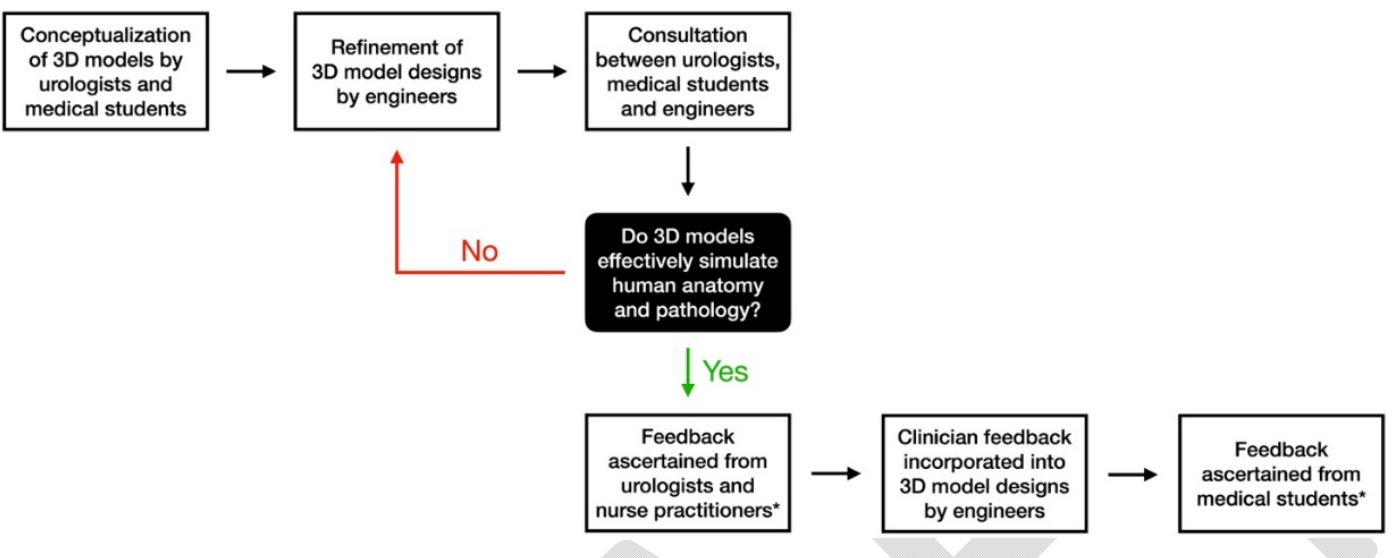

Fig. 2. Photographs of series of 3D- printed testicular cancer models. (A) Represents all five of the series of testicular cancer models. Scrotums depicted contain either, two healthy testicles, or a healthy testicle and a testicle containing an endophytic lesion of varying size. (B) Represents one of the testicular cancer models with stand for easy palpation. (C) Represents the testes from the testicular cancer model. Malignancies are endophytic and cannot be distinguished visually.

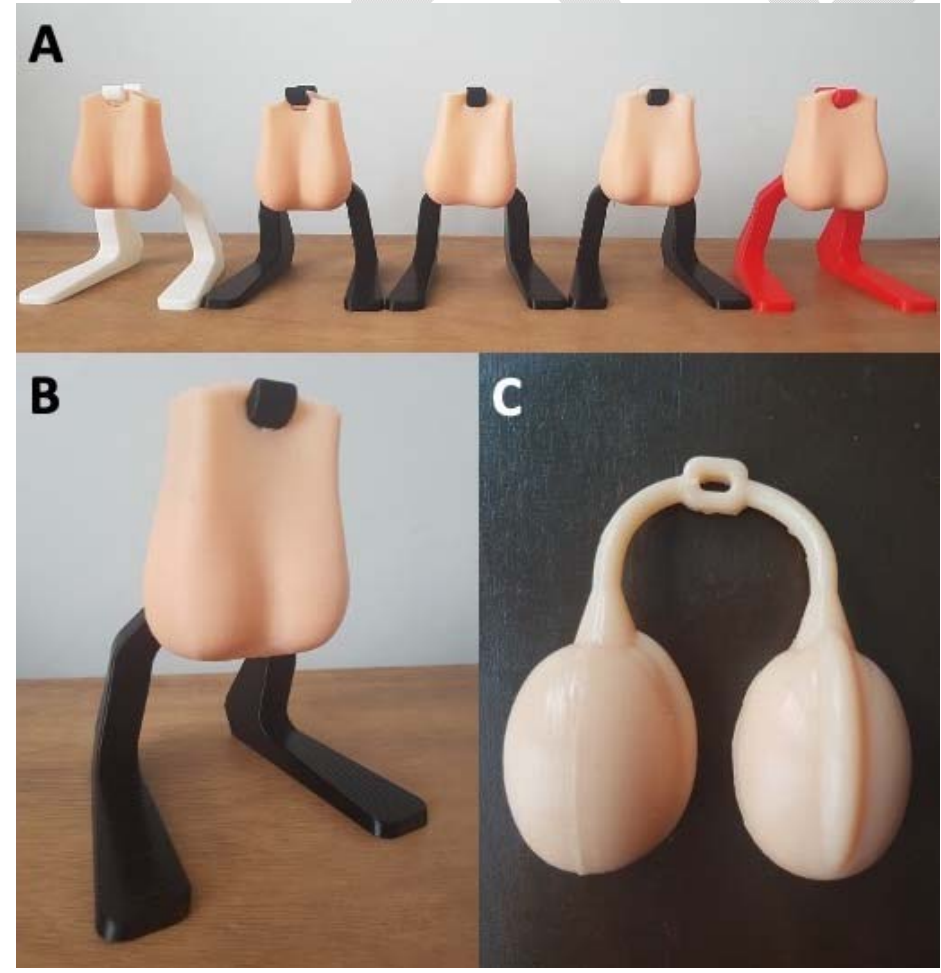




\begin{tabular}{|c|c|c|c|c|c|}
\hline $\begin{array}{l}\text { Questions on model } \\
\text { evaluation survey }\end{array}$ & $\mathbf{n}$ & Mean \pm SD & $\%$ agree* & $\%$ neutral & $\begin{array}{c}\% \\
\text { disagree } \dagger\end{array}$ \\
\hline $\begin{array}{l}\text { This model is anatomically } \\
\text { accurate }\end{array}$ & 8 & $4.5 \pm 0.5$ & 100 & 0 & 0 \\
\hline $\begin{array}{l}\text { On palpation, the testicle with } \\
\text { no mass feels like an accurate } \\
\text { representation of a healthy } \\
\text { testicle }\end{array}$ & 8 & $4.6 \pm 0.5$ & 100 & 0 & 0 \\
\hline $\begin{array}{l}\text { On palpation, the simulated } \\
\text { testicle pathology feels like an } \\
\text { accurate representation of } \\
\text { pathology requiring further } \\
\text { investigation }\end{array}$ & 8 & & 100 & 0 & 0 \\
\hline $\begin{array}{l}\text { This model allows for an } \\
\text { accurate simulation of a } \\
\text { testicular exam }\end{array}$ & 7 & $4.3 \pm 1.0$ & 71.4 & 28.5 & 0 \\
\hline $\begin{array}{l}\text { This model would be useful } \\
\text { teaching tool for patients who } \\
\text { are learning testicular self- } \\
\text { examination }\end{array}$ & 8 & $4.8 \pm 0.7$ & 87.5 & 12.5 & 0 \\
\hline $\begin{array}{l}\text { This model would be a useful } \\
\text { teaching tool for medical } \\
\text { learners who are learning } \\
\text { testicular examination }\end{array}$ & 8 & $4.8 \pm 0.5$ & 100 & 0 & 0 \\
\hline $\begin{array}{l}\text { This model is an improvement } \\
\text { over existing models for } \\
\text { testicular cancer }\end{array}$ & 7 & $4.6 \pm 0.5$ & 100 & 0 & 0 \\
\hline
\end{tabular}

*Percentage of responses as either agree (4) or strongly agree (5). †ंPercentage of response as either disagree (2) or strongly disagree (1). SD: standard deviation. 


\begin{tabular}{|c|c|c|c|c|c|}
\hline $\begin{array}{l}\text { Questions on model evaluation } \\
\text { survey }\end{array}$ & $\mathbf{n}$ & $\begin{array}{l}\text { Mean } \pm \\
\text { SD }\end{array}$ & $\%$ agree $^{*}$ & $\%$ neutral & \% disagree di $^{\dagger}$ \\
\hline $\begin{array}{l}\text { At the beginning of the session, } \\
\text { I possessed the skills to perform } \\
\text { a testicular examination }\end{array}$ & 32 & $1.8 \pm 0.9$ & 6.3 & 12.5 & 81.3 \\
\hline $\begin{array}{l}\text { At the end of the session, I } \\
\text { possessed the skills to perform a } \\
\text { testicular examination }\end{array}$ & 32 & $4.2 \pm 0.4$ & 100 & 0 & 0 \\
\hline $\begin{array}{l}\text { At the beginning of the session, } \\
\text { I felt confident in performing a } \\
\text { testicular examination }\end{array}$ & 32 & $1.6 \pm 0.9$ & 6.3 & 6.3 & 87.5 \\
\hline $\begin{array}{l}\text { At the end of the session, I felt } \\
\text { confident in performing a } \\
\text { testicular examination }\end{array}$ & 32 & $3.9 \pm 0.4$ & 84.4 & 15.6 & 0 \\
\hline $\begin{array}{l}\text { This model is anatomically } \\
\text { accurate }\end{array}$ & 31 & $4.4 \pm 0.6$ & 96.8 & 3.2 & 0 \\
\hline $\begin{array}{l}\text { This model allows for an } \\
\text { accurate simulation of a } \\
\text { testicular exam }\end{array}$ & 30 & $4.3 \pm 0.5$ & 96.7 & 3.3 & 0 \\
\hline $\begin{array}{l}\text { This model would be a useful } \\
\text { teaching tool for patients who } \\
\text { are learning testicular self- } \\
\text { examination }\end{array}$ & 32 & $4.7 \pm 0.5$ & 100 & 0 & 0 \\
\hline $\begin{array}{l}\text { This model would be a useful } \\
\text { teaching tool for medical } \\
\text { learners who are learning } \\
\text { testicular examination }\end{array}$ & 32 & $4.8 \pm 0.4$ & 100 & 0 & 0 \\
\hline
\end{tabular}




\begin{tabular}{|l|l|l|l|l|l|}
\hline $\begin{array}{l}\text { This model would be a useful } \\
\text { addition to the existing urology } \\
\text { curriculum }\end{array}$ & 30 & $4.7 \pm 0.4$ & 100 & 0 & 0 \\
\hline
\end{tabular}

*Percentage of responses as either agree (4) or strongly agree (5). ${ }^{\dagger}$ Percentage of response as either disagree (2) or strongly disagree (1). SD: standard deviation. 